\title{
ESTRUCTURA DE LAS REDES SOCIALES DE LA POBLACIÓN MAYOR DE 60 AÑOS EN CONTEXTOS URBANOS DE POBREZA, DEL DEPARTAMENTO DEL QUINDÍO
}

\section{STRUCTURE OF SOCIAL NETWORKS IN THE ELDERLY PEOPLE IN POVERTY IN THE DEPARTMENT OF QUINDÍO}

\author{
Leonardo Iván Quintana Urrea ${ }^{1}$, César Augusto Gómez Santos $^{1}$ \\ ${ }^{1}$ Programa de Gerontología de la Facultad de Ciencias Humanas y Bellas Artes de la Universidad del Quindío. \\ Miembros del Grupo de Investigación en Desarrollo de la Facultad.
}

Recibido: Agosto 23 de 2010

Aceptado: Noviembre 22 de 2010

Correspondencia: Programa de Gerontología. Universidad del Quindío, Av Bolivar calle 12 norte Armenia Quindío. Correo electrónico: leonardoivan@yahoo.com

\section{RESUMEN}

El propósito de este artículo es presentar los resultados de la investigación del proyecto Redes sociales de la población mayor de 60 años en contextos urbanos de pobreza del departamento del Quindío que enfatiza en la identificación y análisis de la estructura de las redes sociales y las condiciones de vida que presenta esta población, en relación con su tamaño; distancia física, social y psicológica; frecuencia y tipos de contactos; reciprocidad. Desde estos presupuestos, es posible concluir que las redes sociales del Adulto Mayor Pobre en contextos urbanos, juegan un rol determinante en relación con el apoyo que reciben las personas mayores, de parte tanto de la red familiar como de la no familiar. Los datos demuestran que una gran cantidad de ellos son receptores de algún tipo de ayuda y son excepcionales los casos en que, teniendo vínculos con familiares o con amigos y vecinos, no obtienen apoyo. Lo cual demuestra cómo la solidaridad y el soporte mutuo reemplazan o mitigan la ausencia de los recursos necesarios que les permitan acceder a los bienes y servicios que requieren para subsistir. Se confirma que las mujeres son las que mayor cantidad de apoyo reciben; pero que también son ellas las que más ayudas dan dentro de las redes sociales familiares, las no familiares y la red formal.

PALABRAS CLAVE: Redes sociales, estructura de las redes sociales, población mayor, vejez y pobreza.

\begin{abstract}
The purpose of this paper is to present the results of the research social networks of the population over 60 years old in urban poverty in the department of Quindio that emphasizes the identification and analysis of the structure of social networks and life conditions presented by this population, in relation to their size; physical distance, social and psychological distance, frequency and types of contacts, and finally reciprocity. From these premises, it is possible to conclude that social networks of the poor elderly in urban settings play a determining role in relation to the support to the old people, both from the family and the unfamiliar network. The data show that a lot of them are receiving some help and are exceptional the cases where, having ties with family or friends and neighbors, the elderly get no support. This shows how the solidarity and mutual support replace or mitigate the lack of necessary resources that allow them access to goods and services they require to survive. This study confirms that women receive the most amount of support, but they also give so much support to formal and informal networks.
\end{abstract}

KEY WORDS: Social network, structure of social networks, elderly people, old age and poverty.

\footnotetext{
Este artículo surge del proyecto Redes sociales de la población mayor de 60 años en contextos urbanos de pobreza del departamento del Quindío, financiado por la Vicerrectoría de Investigaciones de la Universidad del Quindío, bajo el código de registro 370. Este proyecto hace parte del Grupo de investigación en Desarrollo, adscrito al Programa de Gerontología de la Facultad de Ciencias Humanas y Bellas Artes.

${ }^{2}$ También se puede encontrar la sigla A.M., que corresponde a Adulto Mayor y A.M.P., a Adulto Mayor Pobre.
} 


\section{INTRODUCCIÓN}

Las redes sociales han empezado a tener un interés particular en torno a las problemáticas que presentan las personas mayores de 60 años, y la estructura de estas, pues son la clave para entender las relaciones que se dan entre las redes y las personas en relación con la capacidad para brindar un apoyo social efectivo; además es claro que la investigación sobre ellas y en especial en las comunitarias, no está desarrollada lo suficiente y no existe mucha evidencia sobre el papel que cumplen para la población adulta mayor. Por esto hay que explicar adecuadamente cómo es la dinámica, la estructura y el funcionamiento de las redes sociales de los más viejos, lo que motivó la formulación del problema de investigación:

- Cuál es la estructura y funcionamiento de las redes sociales de las personas mayores de 60 años, en contextos urbanos de pobreza, en el Departamento del Quindío, durante el periodo 2008-2009?

Es importante que se tenga entonces claridad de cómo están estructuradas las redes sociales en la etapa de la vejez; por esto la investigación tuvo entre sus objetivos: Analizar la estructura de las redes sociales de las personas mayores de 60 años, en relación con las condiciones de vida en contextos urbanos de pobreza del Departamento del Quindío, e identificar y establecer la estructura de las redes sociales a las que tienen acceso las personas mayores de 60 años, en relación con su tamaño; distancia física, social y psicológica; frecuencia, tipos de contactos; y reciprocidad.

\section{ANTECEDENTES}

En el plano gerontológico internacional, el tema de las redes sociales no ha sido trabajado tan ampliamente como se desearía; no obstante, en un ámbito más general, se cuenta con algunos desarrollos teóricos importantes que han dado origen a una variada disponibilidad de clasificaciones para identificar los diferentes tipos de redes sociales; también se ha señalado que estas redes se transforman o sufren modificaciones a lo largo del transcurso de la vida de las personas y que existen diferencias significativas cuando de hombres o de mujeres se trata.
La literatura advierte que las redes sociales son importantes para toda la población en general en las diferentes etapas de la vida e incluyen dimensiones que trascienden: la familia con la que se reside, la familia extensa no corresidente, los amigos de barrio o de trabajo, los vecinos, entre otros (Hogan y Eggeben, 1995; Himes y Reidy, 2000). Otros autores han planteado que las redes sociales no solo aluden a los vínculos creados por y para los individuos, sino también a aquellos que son generados de manera grupal o colectiva, tales como la red de redes o las redes comunitarias con las que ha trabajado Elina Dabas (1998) o a las redes de familia que plantea Elizabeth Bott (1990).

El concepto de red social ha sido definido y analizado con variaciones conceptuales propias de las diferentes disciplinas, por ejemplo: Lomnitz (1975) analiza las redes sociales en contextos de pobreza y las define como el conjunto de relaciones de intercambio recíproco de bienes y servicios en un espacio social determinado. La autora hace una diferenciación importante en cuanto a tipos de redes sociales. En primer lugar, define la red egocéntrica que se refiere al total de individuos con quienes ego intercambia recíprocamente bienes y servicios; en segundo lugar, define la red exocéntrica que implica el intercambio entre los distintos vínculos que conforman la red (todos con todos). Esta clasificación le otorga una mayor intensidad y duración a las redes exocéntricas que a las egocéntricas.

González de la Rocha (1986) aborda la clasificación de la red social en cuanto a las características relacionales de los individuos, en términos de jerarquía. Las redes horizontales o de "bordes borrosos" (Dabas, 1998) están principalmente sustentadas en relaciones de parentesco y de amistad y se desarrollan en condiciones sociales y económicas de vida homogéneas. Las redes verticales o de "bordes definidos" (Dabas, 1998) se sustentan en vínculos que el individuo, la familia o un grupo social concreto establece con los sectores formales de la sociedad.

Para Lomnitz (1975), así como para González de la Rocha (1986), Bazán (1998), entre otros, en las redes sociales existen factores determinantes que regulan la efectividad e intensidad de los vínculos. Ellos son la distancia social y su referente sociocultural, la distancia 
física y económica entre los vínculos y su relación con la reciprocidad $y$, por último, la distancia psicológica (confianza y grado de intimidad) entre los individuos que conforman la red social.

Por otro lado, es importante mencionar que la investigación en este campo ha enfatizado fundamentalmente en el apoyo que brindan las redes familiares, las transferencias generacionales y los tipos de ayuda, los niveles de reciprocidad entre padres e hijos, así como las diferencias de género en la recepción de ayuda. Tal pareciera, como lo plantea Montes de Oca (2002) que, tras la investigación sobre envejecimiento, existe el supuesto implícito que inscribe la pasividad en la recepción de ayuda de las personas mayores, porque se les considera incapaces de buscar recursos propios o de salir del ámbito familiar para gestionar la satisfacción de sus necesidades y porque tampoco se les asigna un papel en la comunidad. Esta imagen del adulto mayor provoca que se les considere solo en relación con el ámbito familiar y que casi no haya discusión sobre su papel en la comunidad.

En lo que atañe a Colombia son muy pocos y esporádicos los trabajos relacionados con el tema de las redes sociales, se puede correlatar el trabajo titulado "Relación entre la pertenencia a redes sociales y la dinámica familiar de mujeres en condiciones de pobreza", realizado por Omar Sierra García, Camilo Madariaga Orozco y Raimundo Abello Llanos (1997), en el cual se describe la Red como un mecanismo de supervivencia de estas familias, con incidencia positiva en la dinámica de las mismas.

\section{METODOLOGÍA}

\section{Tipo de estudio}

Este estudio se realizó bajo el enfoque empírico analítico y es una investigación de tipo descriptiva. Buscó aportar en la descripción, explicación y análisis de un fenómeno de importante envergadura en la sociedad colombiana como es el tema de las redes sociales en contextos de pobreza, a partir de observaciones y análisis críticos en el ambiente natural, en el mismo contexto donde sucede. La población se constituyó, a partir de los adultos mayores de 60 años que han sido identificados como pobres (clasificados en el nivel 1 ó 2), mediante el Sistema de Selección de
Beneficiarios (SISBEN 2005 ${ }^{3}$ ), de esta base de datos se tomarón los sujetos, mediante un muestreo probabilístico estratificado, aleatorio, por municipios, y se escogió un número representativo de sujetos de cada uno de ellos, proporcional a la población total de cada municipio.

Se decidió trabajar con un tamaño de muestra para poblaciones infinitas de 591 individuos, con un error muestral del $4 \%$ y una confiabilidad del $95 \%{ }^{4}$. La muestra es de tipo probabilística porque se definió a priori la probabilidad de la selección de las unidades de muestreo. En cuanto al aspecto multietápico, de acuerdo con la estratificación previamente realizada, se eligió un número determinado de sectores, posteriormente se escogieron barrios, luego manzanas y finalmente los hogares (cada uno de los procedimientos se realizó de manera aleatoria).

La selección de los sujetos se realizó mediante un muestreo probabilístico estratificado aleatorio, el tamaño de la muestra fue de 591 personas mayores de 60 años, de los cuales 233 eran hombres 358 eran mujeres cuyas edades oscilaron entre los 60 y los 84 años. De estos 591 se obtuvo que la red no formal está compuesta por 1704 sujetos y 584 componen la red formal. El error muestral fue de 0.04 , esto quiere decir que existe un error potencial del $4 \%$, la confiabilidad de la muestra es del $95 \%$.

Cuadro 1. Población de 60 y más años (Niveles 1 y 2 SISBEN) 2006

\begin{tabular}{lc}
\hline \multicolumn{1}{c}{ MUNICIPIO } & TOTAL \\
\hline ARMENIA & 5385 \\
BUENAVISTA & 147 \\
CALARCA & 4235 \\
CIRCASIA & 1486 \\
CORDOBA & 306 \\
FILANDIA & 506 \\
GENOVA & 547 \\
LA TEBAIDA & 2371 \\
MONTENEGRO & 2750 \\
PIJAO & 350 \\
QUIMBAYA & 2296 \\
SALENTO & 331 \\
TOTAL & $\mathbf{2 0 7 1 0}$ \\
\hline
\end{tabular}

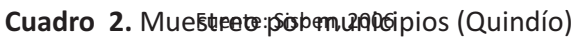

\footnotetext{
${ }_{3}^{3}$ Proporcionada por el Observatorio Social del Departamento del Quindío.

${ }^{4}$ Este procedimiento fue asesorado por el Doctor Hernando Hurtado Tobó. Licenciado en Matemática y física con Maestría en Estadística - Matemática, Director del Grupo de Investigación y Asesoría Estadística de la Universidad del Quindío.
} 
sus preocupaciones $(51,17 \%)$, sus temores $(29,75 \%)$ y sus necesidades $(44,01 \%)$; esta situación podría sugerir que existe una gran distancia psicológica en la red familiar del adulto mayor; sin embargo, si se observan los datos relacionados con las expresiones de confianza y consuelo, éstos indican lo contrario. Por esta razón, los investigadores consideran que esta distancia podría derivar de dificultades en la comunicación o a que los A.M. se abstienen de expresar sus necesidades para evitar malestar a sus familiares, dado que es muy probable que ellos también presenten condiciones de pobreza y no puedan activar el flujo de apoyos que se requiere.

\section{Estructura de la red informal no familiar}

Si los resultados presentados arriba, en relación con la estructura de la red familia, causan preocupación por varios aspectos: redes sociales reducidas, resultados preocupantes en relación con la red informal no familiar, constituida por amigos y vecinos con los cuales se intercambian bienes y servicios. El total de los adultos mayores que conformaron la muestra (591) tienen conjuntamente una red no familiar que escasamente alcanza 584 miembros entre amigos y vecinos, lo cual da como resultado que el tamaño promedio de dicha red ni siquiera alcanza a un miembro (0.99\%).

Llama poderosamente la atención el hecho de que más de la mitad del total de la muestra, $(52,62 \%)$ de los adultos mayores pobres, consideran que no tienen ni un solo vínculo o intercambio con amigo o vecino alguno, mientras que el 23,01\% dijo tener un sólo amigo, seguido por el $11,51 \%$ que expresó que su red informal no familiar la constituían dos amigos; otros datos demuestran que el $6,09 \%$ y $4,23 \%$ de los A.M. pobres tienen redes no familiares constituidas por 3 y 4 amigos respectivamente.

El reducido tamaño de las redes sociales no familiares de los A.M. podría explicarse a partir de aspectos relacionados con el contexto social y económico en el que viven las personas mayores, sujetos de este estudio, con el pasar del tiempo los adultos mayores, en general, enfrentan eventos vitales que le han significado una reducción importante de contactos sociales que se construían a partir de algunos roles también sociales: la jubilación, por ejemplo, implica la mayor posibilidad de perder contactos con antiguos compañeros de trabajo; otros no han logrado acceder a la jubilación pero ya no realizan actividades de carácter productivo, lo que también limita los contactos; a muchos de ellos, la soltería o la viudez les quita la posibilidad de ampliar su círculo de contactos sociales; otros han visto que amigos de muchos años han fallecido y otros más han cambiado de domicilio. Estos, sin ser exhaustivos, son algunos de los eventos que podrían estar condicionando el tamaño de la red. En lo que tiene que ver con el sexo de los miembros de la red no familiar, los datos demuestran que de las 584 personas que la conforman, 408 son mujeres (69.86\%) y 176 son hombres $(30,14 \%)$. En cuanto al tipo de relación que se tiene con los miembros de la red no familiar que brindan apoyo, se encontró que son amigos del A.M., el $57.71 \%$ de sus miembros y vecinos el $38.87 \%$; finalmente, se observa cómo las relaciones con compañeros de trabajo son muy escasas y tan solo representan el $1.54 \%$, a pesar de que el $15,91 \%$, como se mostró en el estatus social del A.M., aún está activo laboralmente.

Cuadro 4. Tamaño de la red no familiar (Vecinos y amigos)

\begin{tabular}{ccc}
\hline Tamaño & TOTAL & $\mathbf{\%}$ \\
\hline $\mathbf{0}$ & $\mathbf{3 1 1}$ & $52,62 \%$ \\
$\mathbf{1}$ & 136 & $23,01 \%$ \\
$\mathbf{2}$ & 68 & $11,51 \%$ \\
$\mathbf{3}$ & 36 & $6,09 \%$ \\
$\mathbf{4}$ & 25 & $4,23 \%$ \\
$\mathbf{5}$ & 5 & $0,85 \%$ \\
$\mathbf{6}$ & 3 & $0,51 \%$ \\
$\mathbf{7}$ & 2 & $0,34 \%$ \\
$\mathbf{8}$ & 1 & $0,17 \%$ \\
$\mathbf{9}$ & 3 & $0,51 \%$ \\
$\mathbf{1 0}$ & 0 & $0,00 \%$ \\
$\mathbf{1 1}$ & 0 & $0,00 \%$ \\
$\mathbf{1 2}$ & 1 & $0,17 \%$ \\
TOTAL & $\mathbf{5 9 1}$ & $\mathbf{1 0 0 , 0 0 \%}$ \\
\hline
\end{tabular}

En la red informal no familiar, se encuentra una correspondencia importante entre el dar y recibir ayudas, tanto en la red familiar como en la no familiar; es decir, el adulto mayor en proporciones similares da y recibe ayuda. Una situación parecida se evidencia en relación con la periodicidad de tiempo con que recibe y da ayuda a la red no familiar: una importante 


\begin{tabular}{lcccc}
\hline & HOMBRES & MUJERES & TOTAL & \multirow{2}{*}{ TOM } \\
\cline { 2 - 3 } & TOTAL & TOTAL & MUESTRA & \\
\hline ARMENIA & 50 & 99 & 149 & $25.21 \%$ \\
BUENAVISTA & 2 & 4 & 6 & $1.02 \%$ \\
CALARCA & 49 & 76 & 125 & $21.15 \%$ \\
CIRCASIA & 14 & 28 & 42 & $7.11 \%$ \\
CORDOBA & 2 & 4 & 6 & $1.02 \%$ \\
FILANDIA & 8 & 4 & 12 & $2.03 \%$ \\
GENOVA & 9 & 9 & 18 & $3.05 \%$ \\
LA TEBAIDA & 26 & 40 & 66 & $11.17 \%$ \\
MONTENEGRO & 32 & 45 & 77 & $13.03 \%$ \\
PIJAO & 8 & 4 & 12 & $2.03 \%$ \\
QUIMBAYA & 27 & 39 & 66 & $11.17 \%$ \\
SALENTO & 6 & 6 & 12 & $2.03 \%$ \\
TOTAL & $\mathbf{2 3 3}$ & $\mathbf{3 5 8}$ & $\mathbf{5 9 1}$ & $\mathbf{1 0 0 \%}$ \\
\hline
\end{tabular}

Técnicas de recolección de la información y tratamiento de los datos

Como técnica de recolección de la información se utilizó el cuestionario, para lo cual se diseñó un instrumento en el que se indagó sobre los indicadores centrales de la estructura y funcionamiento de las redes sociales de apoyo. La información recolectada se procesó a partir de técnicas estadísticas para su cuantificación y análisis; cada una de las variables se trató mediante estadística descriptiva y se hicieron distribuciones de frecuencia y gráficas. Para estos procedimientos se organizó una matriz de datos en el programa estadístico Acces para Windows y se sometió al análisis estadístico propuesto, con paquete Excel.

\section{RESULTADOS}

La investigación en torno a la estructura de las redes sociales de los adultos mayores del departamento del Quindío parte de la descripción de la red familiar desde la perspectiva del adulto mayor; en este sentido, se presenta la percepción de apoyo recibido y brindado que tiene el adulto mayor de su red informal (familiares y amigos) y de su red formal. También se recuerda que esta investigación se realizó desde un análisis de la estrucutura de las redes sociales desde el punto de vista de las redes egocéntricas ${ }^{5}$.

\section{La estructura de la red informal familiar}

Los resultados que se presentan en este articulo obedecen a la percepción del la persona mayor, no se ha indagado el punto de vista ni de los familiares, ni de los amigos que conforman la red informal.

La red familiar de las personas, sujetos de esta investigación, presenta un tamaño promedio de 2,9 miembros por adulto mayor, lo que demuestra que las redes de estos individuos son pequeñas, dado que en relación con los datos del tamaño de la red familiar y la cantidad de familiares de los cuales reciben apoyo, vivan o no con el adulto mayor, resalta el tener al menos 2 familiares; lo que corresponde al $24.87 \%$, seguido muy de cerca por un familiar, lo que equivale al 24.53\%; sin embargo, se evidencian redes de 3 o 4 personas con porcentajes de $17.09 \%$ y $10.83 \%$.

La edad promedio de familiares que componen la red del adulto mayor pobre que reside en la zona urbana es de 42 años. Estos miembros son, a su vez, los que mayor cantidad de apoyo de diversa índole proveen al adulto mayor. Los rangos de edad más representativos son los de 40 a 49 , con un $21.48 \%$ y de 30 a 39 con un $16.84 \%$; esto posiblemente se debe a que en estos rangos de edad, sus miembros, laboralmente activos, pueden brindar con mayor facilidad, apoyo emocional yeconómico.

En cuanto al sexo, se ha encontrado que 938 de los 1704 sujetos que conforman la red familiar de los encuestados corresponde a mujeres que representan el $55.04 \%$ y 766 son hombres con un porcentaje de 45.95\%. Esta distinción es importante porque ha quedado demostrado que en el momento de ofrecer ayuda, son las mujeres las que más dispuestas están a prestar asistencia o apoyo a los miembros más viejos de la red.

Cuadro 3. Tamaño de la red familiar

\begin{tabular}{ccc}
\hline Tamaño & TOTAL & $\%$ \\
\hline $\mathbf{1}$ & 145 & $24.53 \%$ \\
$\mathbf{2}$ & 147 & $24.87 \%$ \\
$\mathbf{3}$ & 101 & $17.09 \%$ \\
$\mathbf{4}$ & 64 & $10.83 \%$ \\
$\mathbf{5}$ & 52 & $8.80 \%$ \\
$\mathbf{6}$ & 26 & $4.40 \%$ \\
$\mathbf{7}$ & 12 & $2.03 \%$ \\
$\mathbf{8}$ & 8 & $1.35 \%$ \\
$\mathbf{9}$ & 7 & $1.18 \%$ \\
$\mathbf{1 0}$ & 4 & $0.68 \%$ \\
$\mathbf{1 1}$ & 1 & $0.17 \%$ \\
$\mathbf{1 3}$ & 1 & $0.17 \%$ \\
$\mathbf{1 5}$ & 1 & $0.17 \%$ \\
NINGUNO & 22 & $3.72 \%$ \\
TOTAL & $\mathbf{5 9 1}$ & $\mathbf{1 0 0 . 0 0 \%}$ \\
\hline
\end{tabular}

Rev. Invest. Univ. Quindío (21): 120-129. Armenia - Colombia 


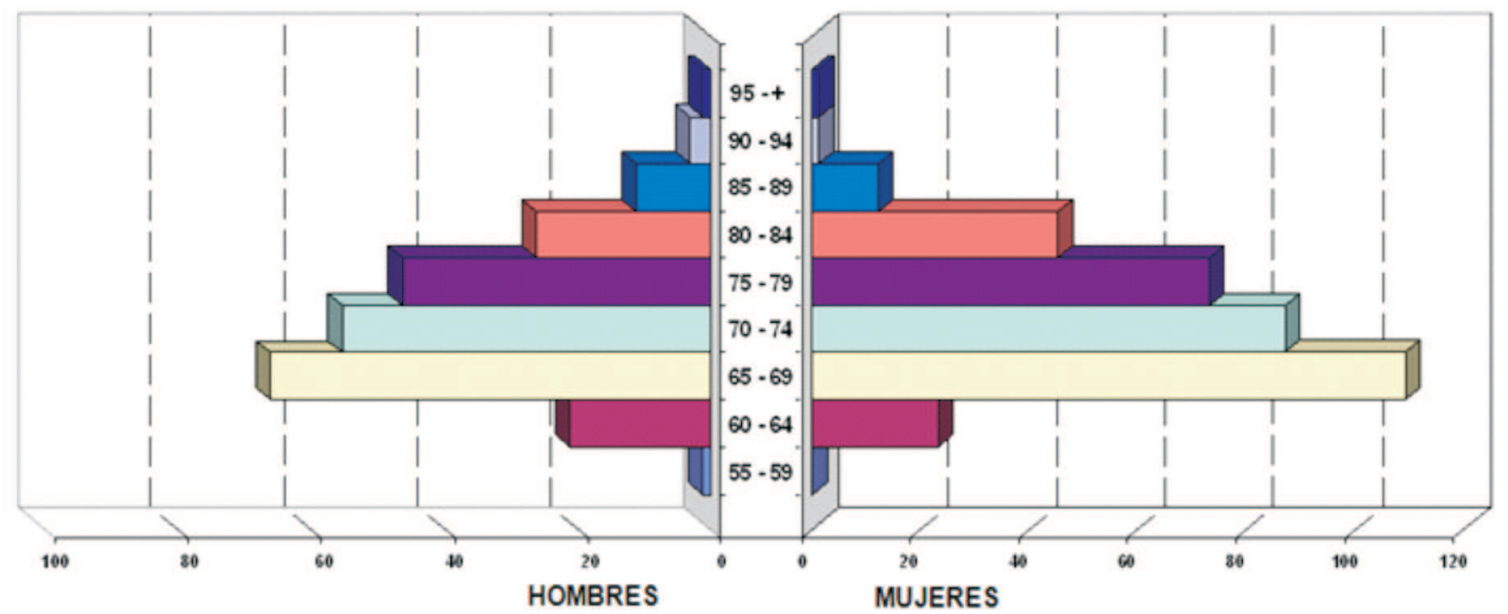

Gráfica 1. Población por grupos de edad y sexo.

En relación con la distancia social (homogeneidad / heterogeneidad) de los miembros de la red familiar con el A.M., determinado por el parentesco, se encontró que los más cercanos son sus hijos con un $59.10 \%$, seguido por los nietos con un $14.03 \%$; este dato resalta como los nietos se convierten en una fuente de apoyos de tipo afectivo o emocional; porque a su temprana edad, éstos aún no hacen parte de la fuerza productiva, lo que les impide brindar otro tipo de ayuda.

En cuanto al estado civil de los miembros de la red familiar que brinda apoyo, se ha encontrado que el $36,27 \%$ están casados y el $36,21 \%$ son solteros; estos porcentajes similares demuestran que el apoyo familiar se da independientemente del estado civil de la persona. Un análisis por sexo permite constatar esta afirmación al verificar que el $43,34 \%$ de los hombres solteros son proveedores de ayuda, en contraste con el $30,38 \%$ de las mujeres también solteras; en este caso, es probable que por no tener una familia, los miembros solteros puedan hacer aportes de carácter material a sus familiares adultos mayores o que cohabiten con ellos en la misma vivienda. Con los miembros casados de la red familiar ocurre lo contrario, se aprecia que los de sexo femenino brindan ayuda en el $39,77 \%$ de los casos, mientras que los de sexo masculino lo hacen en el $31,98 \%$ del total de hombres casados.

De los niveles de escolaridad de los miembros de la red familiar que brindan apoyo a los A.M., el más representativo es la secundaria completa con un $21.54 \%$, le sigue muy de cerca, el nivel de primaria incompleta con un $21.07 \%$ y la primaria completa con un $17.49 \%$, esto redunda en la importancia que hace unos años adquirió al menos tener la secundaria completa para poder acceder al mercado laboral.

Dentro de la estructura de la red social, es importante identificar la frecuencia de las ayudas o apoyos que recibe el adulto mayor de parte de su red familiar: diariamente, en el $59 \%$ de los casos, mensualmente, en el $12 \%$ y semanalmente, en el $10 \%$. Algo significativo para resaltar es que el adulto mayor no es simplemente un receptor pasivo de ayuda de parte de su red familiar, sino que éste también provee ayuda de manera sistemática y, en una gran proporción, casi permanente, a la gran mayoría de los miembros de su familia; así lo indica el hecho de que el $61,50 \%$ de los A.M., proporcionan ayuda a uno o a varios de los miembros de su red familiar con una frecuencia diaria; el $6,57 \%$ semanal y el $3,29 \%$ mensual.

Un alto porcentaje de los miembros de la red familiar del A.M. (el 54,46\%), comparten la vivienda con éste (viven con el A.M.), aspecto que no solamente, se constituye en sí mismo como un apoyo, sino que facilita los intercambios de ayudas entre ellos otro $20,07 \%$ reside en la misma ciudad, el $11,58 \%$ viven en otras ciudades diferentes y un $4,17 \%$ reside en otro país, otras proporciones menos importantes desde el punto de vista porcentual lo constituyen el $4,68 \%$ de los miembros de la red familiar del adulto mayor que vive en el mismo barrio y $3,17 \%$ reside en la misma cuadra.

Contrario a lo que podría esperarse, unas proporciones relativamente bajas de los adultos mayores expresaron a los miembros de su red familiar 
proporción $(39,73 \%)$ recibe apoyo diario de parte de la red informal mientras que el $36,13 \%$ brinda apoyo diario a los miembros de la red familiar; el 16,95\% recibe ayuda semanalmente y el $15,07 \%$ brinda ayuda semanal; el $4,11 \%$ recibe ayuda cada mes mientras que el 2,40 brinda ayuda cada mes, y finalmente el $29,11 \%$ recibe ayuda de manera eventual.

Cuadro 5. Sexo miembros de la red no familiar

\begin{tabular}{lcc}
\hline \multicolumn{1}{c}{ Sexo } & TOTAL & $\%$ \\
\hline 1. Hombre & 176 & $30,14 \%$ \\
2.Mujer & 408 & $69,86 \%$ \\
TOTAL & $\mathbf{5 8 4}$ & $\mathbf{1 0 0 \%}$ \\
\hline
\end{tabular}

A pesar de que en el ámbito familiar se reporta recibiry brindar mayor proporción de ayuda que en el no familiar, es innegable que la solidaridad entre amigos y vecinos permite hacer intercambios importantes como una alternativa distinta a los intercambios entre parientes, que ayuda, en muchos casos, a satisfacer necesidades de primera mano del A.M. que no pueden ser resueltas por la familia.

En cuanto a la distancia física entre el A.M. y los integrantes de la red informal no familiar se encontró que el $44,01 \%$ de ellos vive en la misma cuadra en la que este reside; el $27,40 \%$ de los miembros de la red no formal vive en el mismo barrio en el que vive el A.M.; el $21,40 \%$ en la misma ciudad; $1,88 \%$ en otra ciudad y el $3,42 \%$ comparten su casa con algún amigo. Los datos presentados demuestran que la gran mayoría de los miembros de la red informal de estos están relativamente cerca de él (en su misma casa, cuadra, barrio o ciudad).

Dos variables asociadas a la distancia psicológica entre los miembros de la red no familiar son la confianza expresada entre ellos y la búsqueda de consuelo en los momentos de crisis. A partir de los datos se evidencia la trascendencia que juega la red no familiar (amigos y vecinos), en la vida diaria de los adultos mayores en condición de pobreza, pues en cuanto a la confianza expresada, se encontró que el $74.83 \%$ considera que los vínculos establecidos son de un nivel de confianza tal que permite realizar intercambios de bienes y servicios. De la misma forma, el $69.35 \%$ aceptó buscar consuelo, en los momentos de crisis en los miembros de la red no familiar, en circunstancias en las que la red familiar no se activa.

\section{CONCLUSIONES}

El hecho de que las personas que conformaron la muestra pertenezcan a los niveles 1 y 2 del SISBEN, equivalente a condición de pobreza y pobreza extrema, respectivamente, constituye la razón fundamental por la que los A.M. presentan condiciones de vida que se caracterizan por su precariedad o, lo que es lo mismo, un bajo perfil social; por esta razón, vale la pena resaltar que este perfil se caracteriza por tener una muy baja escolaridad, con una mayor incidencia de mujeres que de hombres; la mayoría $(86,29 \%)$ tiene o tuvo una relación de pareja que le permitió crear descendencia; además, hay presencia de un alto nivel de viudez (mayor en mujeres que en hombres), una relativa baja participación en la fuerza productiva, un muy bajo nivel de acceso a pensión y la existencia de situación de cohabitación con otros miembros de la familia, como hijos, nietos y cónyuges, en su orden.

La estructura de la red informal del adulto mayor pobre (A.M.P) constituida por familiares, amigos y vecinos, se caracteriza por presentar un tamaño promedio de 2,9 personas (en la red familiar), y de $0,99 \%$ (en la red no familiar), esto indica que la red social del adulto mayor es pequeña, lo cual es probatorio de que el comportamiento del envejecimiento demográfico de las poblaciones está afectando negativamente la estructura y funcionamiento de las redes sociales de los viejos, reduciendo las posibilidades de apoyos que podrían recibir los A.M. en contextos de pobreza. Por esta razón, se concluye que estas redes son poco densas y muy cerradas, dado que sus contactos son limitados y, en muchos casos, inexistentes, lo cual obstaculiza la superación de las difíciles condiciones y la satisfacción adecuada de necesidades, a partir de la solidaridad de la red, la limitada interacción implica baja movilidad y pocas posibilidades de potenciar nuevos apoyos y bajos niveles de acción.

En cuanto al tamaño de la red de apoyo se encuentra en la literatura que este no es constante en todas las etapas de la vida, debido a que está determinada en el caso de los adultos mayores por la mortalidad de los contemporáneos, los cambios de domicilio, la pérdida de la pareja o la jubilación. Situaciones a las que se les suman las condiciones geográficas y demográficas de los contextos específicos. 
Algunos antecedentes sobre el tema muestran que en Argentina, el tamaño promedio es de 8.8 personas según Arias (2002), al igual que en ciudad de México, Ham Chande, et al (2002); aunque por otro lado Lomnitz (1994) mostró que en el caso mexicano el tamaño promedio de la red de una familia nuclear alcanzaba cerca de 16 personas. Lo anterior supone que las redes de apoyo de los adultos mayores son más reducidas que en anteriores etapas de la vida. Aunque se pone de manifiesto que no existe un consenso respecto el tamaño de la red de los adultos mayores.

Los resultados de este estudio en relación con el tamaño de la red familiar contrasta con los resultados del estudio de Sierra et al (1997), que se realizó en Barranquilla en donde el tamaño de la red familiar resultó tener en promedio 13 vinculos por mujer trabajadora en condición de pobreza, estos investigadores consideran que estas diferencias se deben a que se estudian poblaciones con características diferentes (edad de los sujetos) y a los aspectos de tipo cultural (población costeña vs población de zona andina).

Los datos presentados corroboran lo que plantea De la Peña (2001), que en cierta medida el que exista una red es muestra de que no hay igualdad de oportunidades para todos los grupos sociales y es en la búsqueda de beneficios de los más desposeídos, donde las redes se estructuran y reestructuran para conservar o aumentar los recursos.

Tanto la red social familiar como la no familiar (amigos y vecinos) está dispuesta cerca al domicilio del A.M.P., esta situación hace factible la ocurrencia de una importante cantidad de intercambios de bienes y servicios, pues como se ha reportado, una mayor distancia física dificulta el ejercicio de los apoyos, en este caso, esa dispersión no es una característica de estas redes.

El vínculo relacional o parental entre los miembros de la red determina la distancia social entre estos y el A.M.P; al respecto, se encontró que los miembros de la red familiar en su orden la constituyen sus hijos, sus nietos, su cónyuge, sus hermanos y, finalmente, su nuera o yerno. Posteriormente, vendría la red no familiar así: amigos, vecinos, compañeros de trabajo y por último se recurre a la red formal para recibir apoyos institucionales. Otro aspecto que sirve como indicador de distancia social lo constituye el nivel de instrucción, dado que un mayor nivel de formación brindaría mejores posibilidades de acceso laboral; en este caso, se concluye que desde el punto de vista educativo no existe una distancia significativa entre el A.M.P., y el nivel educativo de los miembros de su red familiar, a pesar de que los últimos han alcanzado una formación más alta que el de los primeros.

Hasta cierto punto, es lógico y esperado que los A.M.P., experimenten sentimiento de intimidad y confianza hacia los miembros de su red familiar (distancia psicológica), como efectivamente se constata aquí, lo cual les permite expresar con cierta facilidad las preocupaciones, necesidades y temores que ellos experimentan en los momentos de crisis; no obstante, es importante advertir que en muchos casos se considera que miembros de la red no familiar (amigos, vecinos y compañeros de trabajo) son tan bien calificados en este aspectos por los viejos, como ellos mismos lo hicieron con sus familiares, esto significa que la red social no familiar juega un papel fundamental en el apoyo social que reciben los A.M.P., y que en muchos esta, reemplaza, refuerza o complementa los apoyos de la familiar.

Los contactos del A.M.P. con los miembros de la red familiar se presentan con una muy alta regularidad, la mayoría de ellos hacen contacto diario y semanal; situación similar sucede con los miembros de la red no familiar (aunque en proporción un poco menor). Esta regularidad en los contactos hace que a pesar de lo reducido del tamaño de las redes, se propicien los intercambios y apoyos necesarios. Aspectos ya planteados como la cercanía física, social y psicológica permiten que el tipo de contacto que con mayor regularidad se da en general en la red informal (familiar y no familiar) sea el contacto personal o cara a cara, lo cual contribuye a fomentar mayores niveles de confianza y a propiciar apoyos para cubrir las necesidades de asistencia de la población mayor.

Los datos presentados son contundentes en relación con la función del A.M.P. en el ejercicio del apoyo social familiar y no familiar, se desvirtúa la creencia de que éste es un agente pasivo que se limita simplemente a recibir ayuda de la red familiar y la no familiar, es claro que los viejos son proveedores de ayuda a los demás 
miembros de sus redes, incluso se ha encontrado que en algunos casos, brinda ayuda en mayores proporciones comparado con los apoyos recibidos. Paralelamente, esto permite constatar que existe reciprocidad (dar y recibir) en la red social del A.M.P. del departamento del Quindío.

En este orden de ideas, este ejercicio investigativo permite concluir que las redes sociales del A.M., en contextos urbanos de pobreza, juegan un rol determinante en relación con el apoyo que reciben las personas más viejas, de parte tanto de la red familiar como de la no familiar, los datos demuestran que la gran mayoría de ellos obtiene algún tipo de ayuda y son excepcionales los casos en que, teniendo vínculos con familiares o con amigos y vecinos, no se les brinde ayuda. Lo cual demuestra una vez más cómo la solidaridad y el soporte mutuo reemplazan o mitigan la ausencia de los recursos necesarios para acceder a los bienes y servicios que requieren para subsistir, una cosa es que cuantitativamente el tamaño de la red sea limitado y otra que cualitativamente la red juegue un papel decisivo en el bienestar del viejo pobre.

\section{BIBLIOGRAFÍA}

Arias, C. (2002). Red de apoyo social y bienestar psicológico en personas de edad. Tesis para optar al grado de Magister en Psciología Social. Universidad de Mar del Plata, Argentina.

Aranibar, P. (2001). Acercamiento conceptual a la situación del adulto mayor en América Latina. Centro Latinoamericano y Caribeño de Demografía (CELADE) - División de Población de la CEPAL. Santiago de Chile.

Bazán, L. (1998). El último recurso: Las relaciones familiares como alternativa frente a la crisis. Ponencia preparada para LASA 98, Chicago.

Bott, E. (1990). Familia y red social. Roles normas y relaciones externas en las familias urbanas corrientes. Madrid: Taurus Humanidades.

Dabas, E. (1998). Red de redes. Las prácticas de la intervención en redes sociales. Buenos Aires: Paidós.

De la Peña, G. (2001). Presentación Larissa Adler Lomnitz (9-18). En: Lomnitz Larissa Adler, Redes Sociales cultura y poder. Ensayos de Antropología Latinoamericana. México: FLACSO, Ángel Porrúa.

Giraldo N. N. R., De La Rosa B. J. J. y Fernández, Á. A. (2006). Condiciones de vida de los adultos mayores de 60 años en la ciudad de Armenia, Quindío. Armenia, Universidad del Quindío, Vicerrectoría de investigaciones, programa de gerontología. Agosto de 2006.

Gómez S., C. A. (2007). Vejez y pobreza humana en el departamento del Quindío. Informe de investigación, Universidad del Quindío, Vicerrectoría de Investigaciones, Facultad de Ciencias Humanas y Bellas Artes, Programa de Gerontología. Armenia, Febrero de 2007.

Gómez S., C. A. (2008). Vejez y Pobreza en el Departamento del Quindío. En: Revista de Investigaciones Universidad del Quindío, No. 18. Armenia 2008 (pp. 94-107)

González de la Rocha, M. (1986). Los recursos de la pobreza. Familias de bajos ingresos en Guadalajara, Jal. México. Colegio de Jalisco: CIESAS. 
Ham Chande R, P, Torres A. L, e Ibañez E. (2002). Calidad de vida y redes de apoyo social de las personas en edades avanzadas en la ciudad de México. Comisión económica para América Latina y el Caribe CEPAL. CELADE División de población. Reunión de expertos en redes de Apoyo social a personas Mayores: El rol del Estado, la familia y la Comunidad. Santiago de Chile.

Guzmán José Miguel, Huenchuan Sandra y Montes de Oca Verónica. (2003). Redes de apoyo social de personas mayores: marco teórico conceptual. CELADE-División de Población de la CEPAL Universidad Nacional Autónoma de México, México. Ponencia presentada en el Simposio Viejos y Viejas. Participación, Ciudadanía e Inclusión Social 51 Congreso Internacional de Americanistas Santiago de Chile, 14 al 18 de julio de 2003.

Himes, Ch, y Reidy, E. (2000). The Role of Friends in caregiving. Research in Ageing. Vol:22, No. 4 July. Pp. 315-336.

Hogan, D. P. and Eggebeen D. J. (1995). Sources of Emergency Help and Routine Assistance in Old Age, Social Forces. March, 73(3). pp. 917-936.

Lomnitz, L. (1975) ¿Cómo sobreviven los marginados? México: Editorial Siglo XXI.

Lomnitz, L. (1994). Redes sociales, cultura y poder: Ensayos de Antropología Latinoamericana. México: FLACSOGrupo editorial Miguel Ángel Porrúa.

Montes De Oca, V. (2002). Participación, Organización y Significado de las redes de apoyo comunitario entre hombres y mujeres adultas mayores: Laexperiencia de la colonia de Aragón en la delegación Gustavo A. Madero, $\mathrm{Cd}$. De México. Trabajo presentado en la reunión de expertos en redes de apoyo social a personas adultas mayores: El rol del estado, la familia y la comunidad. CEPAL - CELADE. División de Población. Santiago de Chile.

Sierra García, O., Madariaga Orozco, C. y Abello Llanos, R. (1997). Relación entre la pertenencia a redes sociales y la dinámica familiar de mujeres en condiciones de pobreza. Universidad del Norte. Centro de investigaciones en desarrollo humano cidhummaestria en desarrollo social. Barranquilla, Colombia. 